\title{
Enhanced neutron diagnostics data acquisition system based on a time digitizer and transient recorder hybrid module
}

\author{
R.C. Pereira ${ }^{a}, *$ A. Combo ${ }^{a}$, N. Cruz ${ }^{\text {a }}$, Jorge Sousa ${ }^{a}$, \\ C. Correia ${ }^{\mathrm{b}}$, C. Varandas $^{\mathrm{a}}, \mathrm{S}$. Conroy ${ }^{\mathrm{c}}, \mathrm{J}$. Källne ${ }^{\mathrm{c}}$ \\ a Associação EURATOM/IST Centro de Fusão Nuclear, Instituto Superior Técnico, 1049-001 Lisboa, Portugal \\ ${ }^{\mathrm{b}}$ Dep. de Fisica, Universidade de Coimbra, 3004-516 Coimbra, Portugal \\ ${ }^{\mathrm{c}}$ Department of Neutron Research, Uppsala University, EURATOM-VR Association, Box 525, S-75120 Uppsala, Sweden
}

Available online 23 May 2006

\begin{abstract}
This paper proposes a new transient recorder (TR)-time digitizer (TD) hybrid system to be used to pull out higher performance of presently used techniques for measuring the collimated flux and the spectrum of the neutron emission from fusion plasmas of either deuterium or deuterium-tritium, as well as to apply to new neutron measuring techniques, with demanding specific requirements in plasma physics. This system is presented as a modular design, allowing up to four $200 \mathrm{MHz}, 12 \mathrm{~b}$ acquisition channels to operate in an interleaved way, achieving $800 \mathrm{MHz}$. The module includes a XILINX FPGA and an ADC front-end along with a large memory storage capacity and unique TD capabilities (two channels with 36 ps resolution). The FPGA is able to perform real-time data validation as well as some processing algorithms to obtain physical parameters and module calibration. Processed data will be transferred to a control unit by a gigabit optical link. The system will be able to support multiple front-end modules operating synchronously.
\end{abstract}

(c) 2006 Elsevier B.V. All rights reserved.

Keywords: Data acquisition; Neutron diagnostics; Transient recorder; Time digitizer; ADC interleaved architecture; Real-time systems; Signal processing

\section{Introduction}

Neutron diagnostics (ND) play an important role in plasma physics as neutron emission is an important carrier of information about the state of fusion plas-

\footnotetext{
* Corresponding author. Tel.: +351 239410108 ; fax: +351239829158.

E-mail address: ritacp@ lei.fis.uc.pt (R.C. Pereira).
}

mas. ND at JET ${ }^{1}$ include: (i) total neutron yield rate, $Y_{n}(t)$, provided by flux detectors which do not differentiate between neutrons of different energies and are calibrated relative to certain standards and model computation (i.e., not absolute); (ii) neutron profile data from neutron cameras; (iii) energy distribution of collimated neutron flux by neutron emission spectroscopy (NES). NES presents visible enhancements over the

\footnotetext{
${ }^{1}$ Joint European torus.
} 
past 10 years, for instance, the upgrade magnetic proton recoil (MPRu) and the time of flight optimized rate spectrometers [1-3].

Motivated by ITER $^{2}$ needs, further improvements on neutron diagnostics capabilities at JET are being considered. The aim is to have absolute measurement of $Y_{n}(t)$ at high count rate and $2.5 \mathrm{MeV}$ neutrons identification in either D or DT reacting plasmas. With this purpose, a system named MPR-F combining MPRu spectrometer and flux detectors is being designed in order to have neutron spectrum and flux measurements with improved time resolution over large dynamic range [4]. Regarding flux detectors, there are two major issues to solve, setting the threshold, which defines the minimum energy of neutrons that will be accepted in the data and separation of neutrons from background of extraneous radiation, e.g., neutron/gamma discrimination. For the spectrometers the figure of merit is the count rate, leading to fast and continuous acquisition modules.

A new data acquisition system based on TR-TD hybrid module is being developed with the following characteristics: (i) Amplitude resolution of 10-12 b to cope with the expected signal-to-noise ratio (SNR) of the input pulses; (ii) sampling rate of $50 \mathrm{Msamples} / \mathrm{s}$ to 2 GSPS for accurate pulse shaping; (iii) 1 GB of local memory sufficient to store the pulses at the expected rate, during the experiment duration (seconds or tens of seconds).

\section{System description}

This enhanced ND system is presented as a modular TR-TD data acquisition design optically linked to its control unit, in order to be positioned near the diagnostic (Fig. 1). The MPR-F diagnostic requires at least five of these acquisition modules (20 channels) to be implemented on flux detectors in the 19 channels of the JET neutron cameras.

These TR-TD data acquisition modules are plugged into a controller unit through a gigabit communication link as a substitute for the usual $\mathrm{PCI}^{3}$ or $\mathrm{VME}^{4}$ parallel busses.

\footnotetext{
2 "The way" in Latin.

3 Peripheral component interconnect.

4 VERSAmodule Eurocard.
}

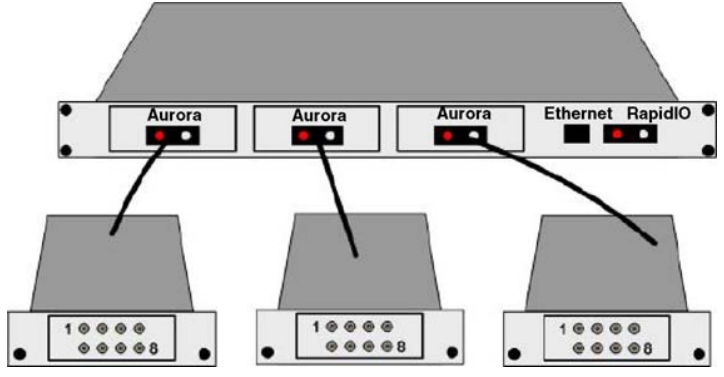

Fig. 1. Enhanced neutron diagnostics hybrid data acquisition system.

The controller module is based on a low power system-on-chip, which includes two PowerPC processors, $512 \mathrm{MB}$ of memory, $8 \mathrm{~Gb}$ links and $10 / 100 \mathrm{Mb}$ Ethernet. The case is a standard $19 \mathrm{in}$. $2 \mathrm{U}$ box with miniITX power supply and forced air ventilation. The operating system will be the LGPL $^{5}$ licensed PowerPC Linux with RTAI ${ }^{6}$ extensions ported to this specific hardware platform. The controller unit will have interconnection to $\mathrm{CODAS}^{7}$ through an Ethernet port. RapidIO interface is also provided for real-time data moving and system monitoring.

Next sections will describe in more detail the TR-TD module.

\subsection{Module architecture}

The TR-TD module is based on FPGA (XCV2P30 from XILINX ${ }^{\mathrm{TM}}$ ) allowing three acquisition architectures, configurable by software: (i) four analogue channels at $200 \mathrm{MSPS}$; (ii) two channels at $400 \mathrm{MSPS}$ based on two interleaved ADCs; (iii) one channel at 800 MSPS based on four interleaved ADCs.

Fig. 2 describes the module architecture with four hybrid input channels featuring $12 \mathrm{~b}$ resolution at $200 \mathrm{MHz}$ sampling rate, for the TR function and $72 \mathrm{ps}$ resolution in a single-chip time to digital converter, achieving 37 ps operating with only two channels. This TD capability permits high accuracy time and amplitude measurements in applications where correlation between both parameters is essential like time-of-flight measurements or coincident trigger discrimination.

\footnotetext{
5 The GNU lesser general public license.

${ }^{6}$ Real-time application interface.

${ }^{7}$ Code ocean data analysis system.
} 


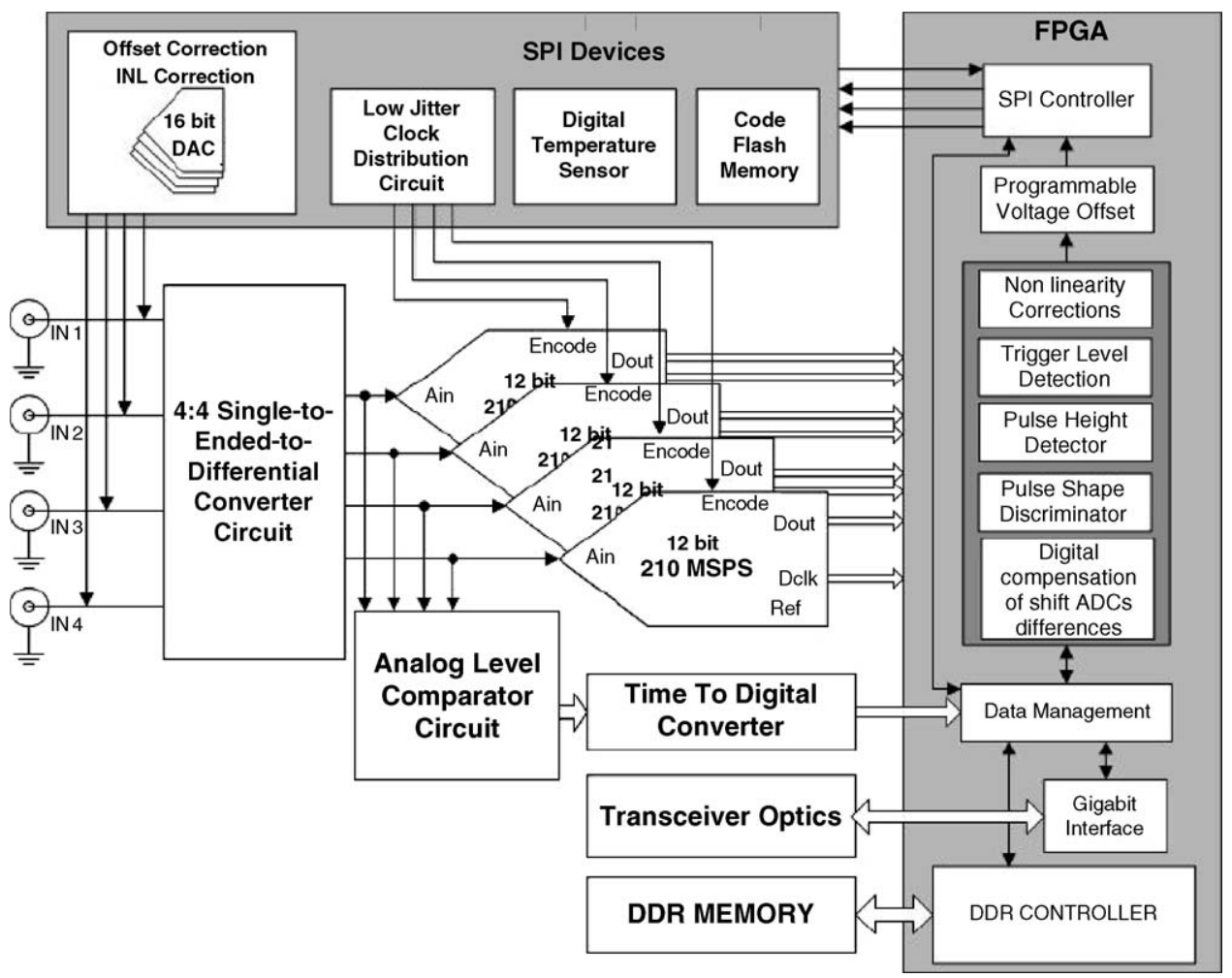

Fig. 2. TR-TD module block diagram.

The FPGA directly connected to the free-running ADC channels performs the following main tasks: (i) analogue inputs calibration; (ii) data storage management; (iii) $3.125 \mathrm{~Gb}$ communications interface; (iv) data processing, including data reduction by pulse height analysis and pulse shape discrimination; (v) complex managing modes of triggering (autotriggering functionality) and finally (vi) serial peripheral interface (SPI) controller. The module provides $1 \mathrm{~GB}$ of double data rate (DDR) synchronous dynamic random access memory.

Interleaved architectures, where channels mismatches are crucial, need temperature sensors to permit calibration of several temperature dependent parameters.

\subsection{Interleaved architecture}

At present, the fastest commercially available $12 \mathrm{~b}$ analogue to digital converter (ADC) is the AD9430, from analogue devices ${ }^{\circledR}$, which is rated to run at
210 MSPS. Faster $12 \mathrm{~b}$ ADCs can be achieved with the already mentioned interleaved architectures, although it represents an immediate threat to the $11 \mathrm{~b}$ dynamic range performance $(68 \mathrm{~dB})$.

At a performance level of $10 \mathrm{~b}$ and above, in interleaved architecture, the dynamic range is dependent on the gain, phase and offset matching between each individual ADC channel. For interleaving mode the following must be noted: (i) the same signal must be attached to each of the $50 \Omega$ input channel connectors of the corresponding interleaved ADCs, the inherent gain mismatches are digitally compensated; (ii) each $\mathrm{ADC}$ has its own internal voltage reference. The possible resultant difference between ADC offsets can be corrected, during module calibration stage, by a $16 \mathrm{~b}$, quad voltage output digital-to-analogue converter; (iii) all ADCs sample at $200 \mathrm{MHz}$, giving an overall data rate of $400 \mathrm{MHz}$ for two channels, with each clock phased at $180^{\circ}$ intervals and $800 \mathrm{MHz}$ for one channel, with each clock phased at $90^{\circ}$ intervals. 


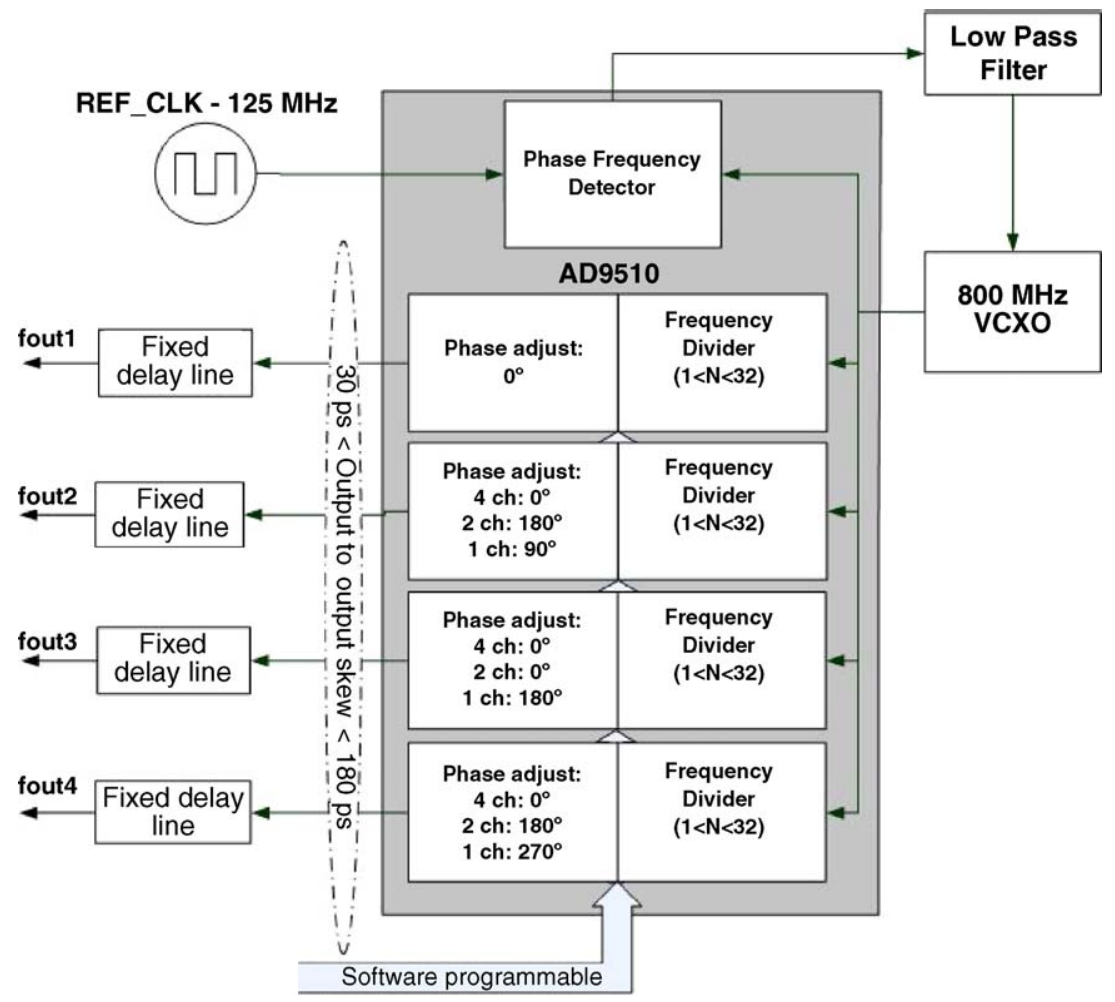

Fig. 3. $800 \mathrm{MHz}$ distribution circuit and clock synthesis.

As ADCs dynamic performance decreases with clock jitter, for this architecture a high quality clock with a maximum rms jitter of $634 \mathrm{fs}$ is needed [5]. Fig. 3 presents the clock distribution circuit exhibiting $355 \mathrm{fs}$ rms jitter (simulated). Fixed delay adjusts were used at each output to fix the clock synthesizer output skew variation (30-180 ps).

\subsection{Data storage}

Each acquired pulse on any channel is tagged and stored with the channel number from where it was generated and the time at which the trigger occurred.

These modules provide a large storage capability of $1 \mathrm{~GB}$ for both raw data (array of sampled pulses) and processed data, which covers all the foreseen scenarios of acquisition time during a JET pulse. Continuous storage of $8 \mathrm{~B}$ at $200 \mathrm{MHz}$ acquisition rate $(1.6 \mathrm{~GB} / \mathrm{s})$ is provided through the interface to the DDR memory that is capable of a maximum of $3.2 \mathrm{~GB} / \mathrm{s}$.

\section{Calibration}

To achieve the desired resolution it is required to calibrate single channel errors and cross channel mismatching. The calibration consists in the calculation of a transfer function of each channel in order to correct the ADC output [6].

The algorithms for obtaining the transfer functions are under study using MATLAB ${ }^{\circledR}$ simulation and include frequency analysis and statistical amplitude measurements, for gain and offset corrections. The aim is to obtain an optimal algorithm to be implemented in the FPGA for on-line calibration.

\section{Data processing}

This system pretends to perform pulse analysis using complex algorithms applied to the digital equivalent of the collected pulses, implemented in the FPGA: 
(i) pulse height analysis, the peak height of the pulse is of prime concern. Using several digital processing algorithms, like moving average, triangular or trapezoidal filter, a histogram of the pulse amplitudes can be done. Pile-up events are also detected and rejected; (ii) pulse shape discrimination, this technique will be based on the pulse area as a shape parameter using the charge integration method. Differences between the integrated charge in the entire pulse and integrated charge over the rising/falling portion are measured. Pulse height and total pulse area can be the same for both neutrons and $\gamma$ rays, but the ratio between the total pulse and the rising/falling portion areas should be different depending on the reaction that took place [7].

\section{Final considerations}

This ND modular data acquisition system will be improved with two more TR modules based on the same interleaved architecture of the above described TR-TD: (i) two channels sampling at $200 \mathrm{MSPS}$ at $14 \mathrm{~b}$ (higher bit resolution at the cost of lower sampling rate); (ii) two channels sampling at 2 GSPS at $8 \mathrm{~b}$ (higher sampling rate at the cost of lower bit resolution) including $0.5 \mathrm{~ns}$ resolution time digitizer.

State of the art modules like for instance the NI PCI5911 high speed digitizer from Analog Devices [8] not only features pulse digitization but also the stamping of the same pulses permitting the random interleaved sampling, increasing the apparent sample rate of repetitive signals. The module presented here features an even more accurate pulse shaping and pulse temporal positioning.

\section{Acknowledgements}

This work has been carried out in the frame of the Contract of Association between the European Atomic Energy Community and Instituto Superior Técnico (IST) and of the Contract of Associated Laboratory between Fundação para a Ciência e Tecnologia (FCT) and IST. The content of the publication is the sole responsibility of the authors and it does not necessarily represent the views of the Commission of the European Union or FCT or their services.

\section{References}

[1] J. Källne, L. Giacomelli, A. Hjalmarsson, S. Conroy, G. Ericsson, M. Gatu Johnson, et al., Advanced neutron diagnostics for ITER fusion experiments, UU-NF 04\#14, December 2004.

[2] A. Combo, R. Pereira, J. Sousa, N. Cruz, P. Carvalho, C.A.F. Varandas, S. Conroy, J. Kallne, M. Weiszflog, A PCI transient recorder module for the JET magnetic proton recoil neutron spectrometer, Fusion Eng. Des. 71 (1) (2004) 151-157.

[3] J. Sousa, A.J.N. Batista, A. Combo, R. Pereira, N. Cruz, P. Carvalho, C.A.F. Varandas, S. Conroy, G. Ericsson, J. Kallne, A PCI time digitizer for the new JET time-of-flight neutron spectrometer, Fusion Eng. Des. 71 (1) (2004) 101-107.

[4] L. Giacomelli, S. Conroy, G. Ericsson, G. Gorini, H. Henriksson, A. Hjalmarsson, J. Källne, M. Tardocchi, Comparison of neutron emission spectra for D and DT plasmas with auxiliary heating, Eur. Phys. J. D 33 (2005) 235-241.

[5] AN-501, http://www.analogdevices.com.

[6] M. Looney, Advanced digital post-processing techniques enhanced performance in time-interleaved ADC systems, Analog Dialogue 37-8 (August) (2003).

[7] V.T. Jordanov, G.F. Knoll, Digital Synthesis of pulse shapes in real-time for high resolution radiation spectroscopy, Nuclear Instrum. Methods Phys. Res. A 345 (1994) 337-345.

[8] NI PCI-5911 specifications, http://www.ni.com. 